\title{
Telemedicine for Asthma Follow-up in Children During COVID-19 Pandemic
}

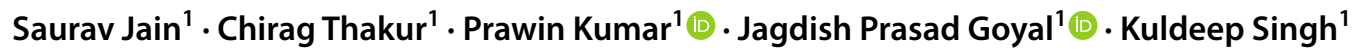

Received: 10 May 2021 / Accepted: 15 June 2021 / Published online: 28 July 2021

(c) Dr. K C Chaudhuri Foundation 2021

To the Editor: Asthma management is a continuous cycle of assessment, treatment, and review by a trained person [1]. However, the coronavirus pandemic has disrupted this continuum of asthma care [2,3]. Telemedicine can provide successful follow-up care for respiratory illnesses in children [4]. We present a retrospective study on telemedicine follow-up of children with asthma at a tertiary care center from western India.

A total of 156 children (boys: 103) with asthma [mean (SD) age 10.6 (3.4) y] were followed up with telemedicine services from April 15 to October 31, 2020. Out of them, $111(71.2 \%)$ children continued their regular inhaled corticosteroids (ICS); 42 (37.8\%), 56 (50.5\%), and 13 (11.7\%) were on step II, III, and IV therapy, respectively, as per the Global Initiative for Asthma [1]. The remaining 45 (28.8\%) children had stopped taking regular ICS; the reasons were no current symptoms $(30 ; 66.6 \%)$, unavailability of medication due to lockdown $(6 ; 13.3 \%)$, migration of parents $(1 ; 2.2 \%)$, and economic issues $(1 ; 2.2 \%)$. In contrast, $7(6.4 \%)$ children were taking ICS intermittently.

Asthma symptoms were well-controlled, partly controlled, and uncontrolled in 137 (87.5\%), 13 (8.5\%), and 6 (3.9\%) children, respectively. Asthma exacerbation occurred in $13(8.3 \%)$ children; $10(6.4 \%)$ responded with only inhaled short-acting $\beta-2$ agonist, while 3 (1.9\%) children also required oral steroids. Video-calling through WhatsApp was performed to assess the inhalation technique in children with partly and uncontrolled asthma $(19 ; 12.1 \%)$, in which $16(84.2 \%)$ children had good technique, while 3 $(15.7 \%)$ had poor technique. Simultaneously, feedback for the correct inhalation technique was given. Partly controlled and uncontrolled asthma was more common in children with poor compliance to therapy [16 (84.2\%) vs. $3(15.8 \%)$;

Prawin Kumar

drprawin484@gmail.com

1 Department of Pediatrics, All India Institute of Medical Sciences, Jodhpur, Rajasthan 342005, India $p<0.001]$. Treatment was restarted in 27 (17.3\%), stepped up in $2(1.3 \%)$, and stepped down in $6(3.8 \%)$ children. Only $86(55.1 \%)$ children were on regular $(\geq 2)$ telemedicine follow-up.

In conclusion, this study has shown that telemedicine is a potential tool in the follow-up of children with asthma that can bridge the gap in the continuum of asthma care during this ongoing pandemic.

\section{Declarations}

Conflict of Interest None

\section{References}

1. Global initiative for asthma (GINA).In: Global strategy for asthma management and prevention. 2021. Available at: https://ginasthma. org/wp-content/uploads/2021/04/GINA-2021-Main-Report_ FINAL_21_04_28-WMS.pdf. Accessed on 7 May 2021.

2. Kumar P, Goyal JP. Management of asthma in children during COVID-19 pandemic. Indian Pediatr. 2020;57:684-5.

3. Papadopoulos NG, Custovic A, Deschildre A, et al. Pediatric asthma in real life collaborators. impact of COVID-19 on pediatric asthma: practice adjustments and disease burden. J Allergy Clin Immunol Pract. 2020;8:2592-99.

4. Kumari J, Jat KR, Kabra SK. Role of telemedicine in followup care of children with respiratory illnesses at a tertiary care hospital - an ambispective observational study. Indian J Pediatr. 2021;4:1-5.

Publisher's Note Springer Nature remains neutral with regard to jurisdictional claims in published maps and institutional affiliations. 